$\mathrm{Da}$ viel Tausch-, Kauf- und Handelsrecht dargestellt wird, liegt angesichts der Stellung des Berichterstatters auf der Hand. Einen breiten Raum nimmt die Schilderung dessen ein, was im weitesten Sinne unter Ehe-, Familien- und Erbrecht einzuordnen ist, nämlich Schilderungen sexueller Praktiken, Hochzeitsrituale, Sippenkämpfe, geregelte Sterbehilfe und die oft grausamen Beerdigungsriten.

Am frendartigsten erscheint dem heutigen Leser natürlich das Rechtsinstitut des Kannibalismus, das in ein Kriegs- und Friedensrecht eingebettet ist. Dieses wieder ist eng verknüpft mit den Männerbünden, die wie in anderen Teilen der Welt, z.B. in Westafrika, wo es nicht zur Staatenbildung gekommen ist, die Ansätze der Ausbildung ziviler Gewalt, hier die Dorfältestenräte, überlagemd als gesellschaftstragende und -regulierende Institution sowohl legislative, exekutive als auch judikative Funktionen haben.

Gegliedert ist das Buch in vier geographische Groß-Abschnitte, entsprechend den Einsatzorten des Berichterstatters, wodurch Rechtsunterschiede und -gemeinsamkeiten deutlich werden. Fast die Hälfte des Buches ist den Witu-Inseln (nicht zu verwechseln mit dem ebenfalls Kaiserlich-Deutschen "Wituland" in Ostafrika, das das eigentliche Tauschobjekt beim Erwerb von Helgoland war, vgl. dazu Hans Peter Ipsen, Helgoland 100 Jahre deutsch, in: DÖV 1990, S. 581-588, besonders S. 583 Fn. 10) gewidmet, einer Inselgruppe, über die bisher so gut wie nichts veröffentlicht worden ist. Im übrigen sind die Berichte zeitlich Vorläufer der späteren Forschungsberichte des bedeutenden Ethnosoziologen Richard Thumwald (1869-1954), der nach Karl Vieweg nach Melanesien kam und sich der Hilfe des kundigen Karl Vieweg bediente, worüber das Buch ebenfalls berichtet.

Der Verfasser hat die 80 Jahre alten, bisher unveröffentlichten Berichte seines Vaters auf einer eigenen Forschungsreise 1988 verifiziert und mit eigenen Erlebnissen, Beobachtungen und Vergleichen von Vergangenheit und Gegenwart angereichert. Dadurch ist ein so material- und detailreiches Werk entstanden, daß es eine wertvolle Bereicherung der historischen Literatur vor allem des auf der Suche nach einer eigenen nationalen Identität befindlichen Staates Papua-Neuguinea ist.

Gerold Schmidt

\title{
Cord-Georg Hasselmann
}

Die Freiheit der Handelsschiffahrt - Eine Analyse der UN-Seerechtskonvention -

Veröffentlichungen des Instituts für Seerecht und Seehandelsrecht der Universität Hamburg, herausgegeben von Rolf Herber und Rainer Lagoni, Band 1.

N. P. Engel Verlag, Kehl am Rhein, Straßburg, Arlington 1987, 507 S., DM 136,-

Die Seerechtskonvention der Vereinten Nationen vom 10.12.1982 (SRK) gilt zu Recht als eine der bedeutendsten völkerrechtlichen Kodifikationen seit 1945. Sie hat nicht nur die 
Genfer Seerechtskonventionen von 1958 weiterentwickelt und bisher lediglich gewohnheitsrechtlich bestehendes Seevölkerrecht kodifiziert, sondern darüber hinaus erstmals rechtliche Regeln für technologisches Neuland (vgl. Art. 133 ff. SRK betr. Tiefseebergbau) erschlossen. Zugleich aber hat die "package deal" zwischen traditionellen Materien des Seevölkerrechts und - häufig in stetem Konflikt zwischen Industrie- und Entwicklungsländern geschaffenem - "neuem Seerecht" bisher ein Inkrafttreten der Konvention verhindert: Nicht zuletzt der Widerstand wichtiger Industriestaaten (wie der Bundesrepublik, Großbritanniens und der USA) gegen u.a. ein als dirigistisch empfundenes Meeresbodenregime hat die für ein Inkrafttreten gem. Art. 308 SRK vorausgesetzte Ratifikation durch mindestens 60 Staaten verzögert. Damit wurde indes nicht nur das ungeliebte Tiefseebergbauregime, sondern auch die Geltung wichtiger Schiffahrtsregeln blockiert. Nachdem sich jedoch der Ratifikationsstand nunmehr der erforderlichen Schwelle nähert (43 hinterlegte Ratifikationsurkunden am 2.5.1990) und auch in der Bundesrepublik - nicht zuletzt wegen der in Art. 1 Annex VI SRK vorgesehenen Ansiedlung des Seegerichtshofes in Hamburg - eine baldige Ratifikation zunehmend wahrscheinlich wird, ist ein Inkrafttreten der SRK innerhalb der nächsten Jahre zu erwarten.

Gleichzeitig wächst damit wieder der Bedarf an Gesamtdarstellungen dieser Konvention in deutscher Sprache. Eine der ersten umfassenden und systematischen Untersuchungen zum Schiffahrtsrecht der SRK stellt dabei die Hamburger Dissertation von C.-G. Hasselmann aus dem Jahre 1986 dar.

Ziel der Untersuchung ist, aufzuzeigen, inwieweit in dem durch die Konvention kodifizierten System an das Schiff anknüpfender Rechte und Pflichten verschiedener Staaten das Interesse der internationalen Handelsschiffahrt an möglichst ungehinderter Bewegungsfreiheit berücksichtigt wurde.

Der Verfasser gibt zunächst eine knappe Einführung in die relevanten Grundlagen des Seevölkerrechts (S. 8 ff.), in deren Rahmen die Beziehung von Schiffahrtsfreiheit und Flaggenhoheit, die Entwicklung des Seerechts und der Verlauf der Dritten Seerechtskonferenz ebenso dargestellt werden wie die Probleme einer Auslegung der SRK bzw. ihrer Stellung im Rechtsquellensystem des Völkerrechts. Es folgt im zweiten Teil eine Klärung des Schiffsbegriffs der Konvention sowie anderer internationaler und nationaler seerechtlicher Regelungen (S. 55 ff.), bevor Hasselmann - der zonalen und funktionalen Gliederung der SRK entsprechend - die Handelsschiffahrt auf der Hohen See (S. 68 ff.), in der Ausschließlichen Wirtschaftszone (S. 109 ff.) und Anschlußzone (S. 228 ff.), im Küstenmeer (S. 244 ff.), in Meerengen (S. 306 ff.), inneren (S. 352 ff.) und Archipelgewässern (S. 365 ff.) sowie - wegen ihrer besonderen Anschaulichkeit für das Verhältnis der Jurisdiktionen von Flaggen- und Küsten- bzw. Hafenstaat, die auch zu einer Verselbständigung dieser Materie im XII. Teil der Konvention geführt hat - die Verschmutzungskontrolle (S. $388 \mathrm{ff}$ ) und abschließend das System der Streitschlichtung (S. $441 \mathrm{ff}$.) untersucht. Nicht berücksichtigt wurden - neben Fragen des Seekriegsrechts bzw. des Status von Kriegsschiffen insbesondere Schiffahrtsfragen im Zusammenhang mit Fischerei (vgl. Art. 61 ff. SRK), Meeresforschung (Teil XIII der SRK) und Tiefseebergbau (Teil XI der Konvention). 
Innerhalb der einzelnen Kapitel schildert der Verfasser nach einer kurzen Einleitung in der Regel die Behandlung der jeweiligen Materie während der Konferenz, bestimmt sodann den räumlichen Geltungsbereich der betreffenden Normen, analysiert den rechtlichen Status der Meereszone sowie die Rechte und Pflichten der Küstenländer und der Schiffe dritter Staaten und schließt mit einer Untersuchung der relevanten Staatenpraxis.

Angesichts der Komplexität der Materie und des umfassenden, handbuchartigen Charakters der Untersuchung seien hier nur einige Schwerpunkte herausgehoben.

Bei der Untersuchung des der SRK zugrundeliegenden Schiffsbegriffs kommt der Verfasser zunächst zu dem Ergebnis, daß dieser sich mangels Definition aus dem jeweiligen Normkontext ergebe (S. 60), zumal auch kein einheitlicher internationaler Schiffsbegriff existiere und eine Úbernahme der divergierenden nationalen Ansätze nicht wünschenswert sei (S. 67); allerdings zieht er nicht die Konsequenz einer nunmehr normorientierten Betrachtung, sondern geht unmittelbar zu der Handelsschiffahrt auf der Hohen See über. Deren Regime hat seit den Genfer Konventionen von 1958 insbesondere durch die Ausdehnung der nationaler Hoheit unterliegenden Küstengewässer und die Verstärkung der dem Flaggenstaat obliegenden Pflichten erhebliche Veränderungen erfahren, die - nach einer Erläuterung von Rechtsstatus und Inhalt von Meeres- und Schiffahrtsfreiheit - im Mittelpunkt dieses Untersuchungsabschnittes stehen. Umrahmt von Ausführungen zu den Geboten der Rücksichtnahme und freundlichen Nutzung (Art. 87 II, 88 SRK), der Strafgerichtszuständigkeit (Art. 97 SRK) und diverser Kooperationspflichten (Art. 98-109 SRK) bilden dabei Ausführungen zur Staatszugehörigkeit der Seeschiffe (Art. 90-93 SRK) den Kern der Erläuterungen. Trotz einiger Ungenauigkeiten (S. 86 f; so war Art. 5 der Genfer Hohe-SeeKonvention weniger eine Reaktion auf die durch die sog. Offenen Register bedrohten Sozial- und Sicherheitsstandards, sondern der Versuch der westeuropäischen Schiffahrtsstaaten, den ausflaggungsbedingten Wettbewerbsvorteil der US-Reeder zu beseitigen, wobei das in Art. 5 GHSK enthaltene Erfordernis eines "genuine link" auch nicht wegen des bekannten IGH-Gutachtens im "IMCO"-Fall zur lex imperfecta wurde, sondern infolge Streichung der ursprünglich vorgesehenen Sanktion - in Form der Nichtanerkennung der Staatszugehörigkeit - im Rahmen der Schlußabstimmung während UNCLOS I) werden Inhalt und Bedeutung von Art. 91, 94 SRK zutreffend analysiert. Gleiches gilt für den Exkurs über Vorgeschichte und Inhalt der UN-Schiffsregisterkonvention von 1986 (S. 89 ff.). Jedoch entgeht dem Verfasser hier, daß die Begriffe vom "economic" bzw. "administrative link" nur Schlagworte darstellen für den dahinterstehenden fundamentalen Streit, ob die "genuine link"-Bedingungen vor der Verleihung der Staatszugehörigkeit erfüllt sein müssen oder - sich in dem Pflichtenkatalog des Art. 94 SRK erschöpfend - auch danach erfüllt werden können; ferner ist seine Annahme (S. 93), die Registerkonvention stelle eine wesentliche Verstärkung der Verbindung zwischen Flaggenstaat und Schiff dar, angesichts der Vielzahl in ihr enthaltener höchst unbestimmter Rechtsbegriffe und des mit vielen Schlupflöchern versehenen "optional approach" der Art. 7-9 RegKonv wohl zumindest zweifelhaft. 
Jedoch vermögen diese Kritikpunkte den Wert der Untersuchung im Ergebnis nicht zu schmälern. Hasselmann hat das klassische und praktisch so wichtige Spannungsverhältnis zwischen flaggenstaatlichem und küstenstaatlichem Regelungsanspruch für nahezu alle Stationen, die ein Handelsschiff auf seiner Fahrt durchläuft, untersucht. Seine umfangreichen Nachweise in- wie ausländischer Literatur geben vielfältige Ansatzpunkte für weitere punktuelle Recherchen. Als grundlegende, handbuchartige Einführung in das internationale Schiffahrtsrecht steht "der Hasselmann" damit schon heute im Mittelpunkt der deutschsprachigen Literatur zur Seerechtskonvention.

Marco Núñez-Müller

\section{Nikolaos St. Skourtos}

\section{Die Billig-Flaggen-Praxis und die staatliche Verleihungsf reiheit}

Studien zum Internationalen Wirtschaftsrecht und Atomenergierecht, herausgegeben von V. Götz, G. Gornig, D. Rauschning und G. Zieger, Bd. 83

Carl Heymanns Verlag 1990, 350 S., DM 58,-

$\mathrm{Da} \beta$ auch völkerrechtliche Fragestellungen quasi konjunkturellen Zyklen unterliegen können, läßt sich u.a. anhand des Problems der Staatszugehörigkeit von Schiffen zeigen.

Bereits 1896 normierte das IDI zwecks internationaler Vereinheitlichung der Flaggengesetzgebung einen Katalog völkerrechtlicher Voraussetzungen für Erwerb und Verlust des Flaggenführungsrechts und damit der Staatszugehörigkeit von Schiffen1, der allerdings von den Mitgliedstaaten nicht umgesetzt wurde. Die rasante Entwicklung der sog. "Offenen Register" mit ihren Kostenvorteilen und dem dadurch ausgelösten Ausflaggungsdruck zulasten der westeuropäischen Schiffahrtsnationen nach 1945 machte diese Frage zu einem der Hauptprobleme der 1. Seerechtskonferenz. Heraus kam mit Art. 5 Abs. 1 des Genfer Ubereinkommens über die Hohe See (GUHS) von 19582 einerseits die völkerrechtliche Anerkennung der traditionellen Freiheit der Staaten bei der Kodifizierung ihrer nationalen Flaggenverleihungsvoraussetzungen, andererseits - in Anlehnung an die im "Nottebohm"Urteil des IGH3 zur Parallelproblematik der Staatsangehörigkeit natürlicher Personen entwickelten Konzeption - die berühmte Formulierung

"There must exist a genuine link between the State and the ship; in particular, the State must effectively exercise its jurisdiction and control ...".

1 Règles relatives à l'usage du pavillon national pour les navires de commerce, in: Annuaire de l'Institut de Droit International XV (1986), 1069.

2 UNTS 450, S. 11; BGBl. 1972 II 1089.

3 ICJ Reports 1955, 4 (23). 